\title{
Post-mortem genetic investigation of cardiac disease-associated genes in sudden infant death syndrome (SIDS) cases
}

\author{
Jasmin Köffer ${ }^{1} \cdot$ Stefanie Scheiper-Welling ${ }^{1} \cdot$ Marcel A. Verhoff ${ }^{1} \cdot$ Thomas Bajanowski $^{2} \cdot$ Silke Kauferstein $^{1}$
}

Received: 10 June 2020 / Accepted: 3 August 2020 / Published online: 12 August 2020

(C) The Author(s) 2020

\begin{abstract}
The sudden infant death syndrome (SIDS) is one of the leading causes of postneonatal infant death. It has been shown that there exists a complex relationship between SIDS and inherited cardiac disease. Next-generation sequencing and surveillance of cardiac channelopathy and cardiomyopathy genes represent an important tool for investigating the cause of death in SIDS cases. In the present study, targeted sequencing of 80 genes associated with genetic heart diseases in a cohort of 31 SIDS cases was performed. To determine the spectrum and prevalence of genetic heart disease associated mutations as a potential monogenic basis for SIDS, a stringent variant classification was applied and the percentage of rare (minor allele frequency $\leq 0.2 \%$ ) and ultrarare variants (minor allele frequency $\leq 0.005 \%$ ) in these genes was assessed. With a minor allele frequency of $\leq 0.005 \%$, about $20 \%$ of the SIDS cases exhibited a variant of uncertain significance (VUS), but in only $6 \%$ of these cases, gene variants proved to be "potentially informative." The present study shows the importance of careful variant interpretation. Applying stringent criteria misinterpretations are avoided, as the results of genetic analyses may have an important impact of the family members involved.
\end{abstract}

Keywords Sudden infant death syndrome $\cdot$ SIDS $\cdot$ Genetic heart disease $\cdot$ Next-generation sequencing $\cdot$ Targeted sequencing · Molecular autopsy

\section{Introduction}

The sudden infant death syndrome (SIDS) is still one of the leading causes of postneonatal infants death [1]. SIDS is defined as sudden death of an infant under 1 year of age, and the cause of death still remained unexplained after autopsy [2]. In most cases, death occurs during sleep. The peak incidence is between 2 and 4 months and is higher in male than in female infants [3].

Jasmin Köffer and Stefanie Scheiper-Welling contributed equally to this work.

Electronic supplementary material The online version of this article (https://doi.org/10.1007/s00414-020-02394-x) contains supplementary material, which is available to authorized users.

Silke Kauferstein

kauferstein@em.uni-frankfurt.de

1 Institute of Legal Medicine, University of Frankfurt, Kennedyallee 104, 60596 Frankfurt/Main, Germany

2 Institute of Legal Medicine, University of Essen, Hufelandstraße 55, 45122 Essen, Germany
A "triple risk model" for SIDS has been proposed and implies that the risk of SIDS increases if three factors concur: (1) a vulnerable infant, (2) a critical period in the development during the first months after birth, and (3) exogenous stress factors such as sleeping in prone position, smoking of the parents, and premature birth $[1,4]$. Despite some successful campaigns to reduce these risk factors, SIDS is still the leading cause of death in postneonatal infants [5].

Genetic variants affecting autonomic functions, neurotransmission, energy metabolism, and cardiac repolarization have been suggested to contribute to SIDS [6-9]. These studies also showed that there exists a complex relationship between SIDS and inherited cardiac diseases. Long QT syndrome (LQTS), short QT syndrome (SQTS), Brugada syndrome (BrS), catecholaminergic polymorphic ventricular tachycardia (CPVT), and hypertrophic cardiomyopathy (HCM) have been described as a monogenic cause in a certain number of SIDS cases [6, 9-14].

The development of next-generation sequencing (NGS) offers new opportunities to investigate potential pathogenic sequence variations as an underlying cause of death in SIDS cohorts. Several studies suggest that in up to $30 \%$ of SIDS cohorts pathogenic mutations in cardiac channelopathy- 
associated genes contributed as a monogenic cause of death $[4,9,12,13]$.

In $3.5 \%$ of SIDS cases, a pathogenic variation in a cardiomyopathy-associated gene was detected [14].

The detection of variants and its evaluation is important when counseling the family members in a SIDS case, but special precautions have to be made when a variant is considered to be pathogenic. In a recent study of variant analysis, $4.3 \%$ of pathogenic variations and $13 \%$ of "informative variations" have been found, which is a much lower percentage than that reported in previous studies [2].

In the present study, an analysis of genes associated with genetic heart diseases in a cohort of 31 SIDS cases was performed to investigate the presence of potentially causative variants, which may represent a predisposing risk factor for sudden death in infancy.

\section{Methods}

Study group The SIDS cohort consisted of 31 SIDS cases (Table 1). Pulmonary tissue samples had been collected during autopsy between 1999 and 2001 as part of a study on SIDS (GeSID) by Findeisen et al. [15]. These samples were stored at

Table 1 Summary of the sudden infant death syndrome cohort

\begin{tabular}{lll}
\hline Case & Sex & Age \\
\hline 1 & $\mathrm{~m}$ & 7 weeks \\
2 & $\mathrm{f}$ & 7 weeks \\
3 & $\mathrm{~m}$ & 2.5 months \\
4 & $\mathrm{~m}$ & 3 months \\
5 & $\mathrm{f}$ & 3 months \\
6 & $\mathrm{~m}$ & 1.5 months \\
7 & $\mathrm{f}$ & 2 months \\
8 & $\mathrm{f}$ & 4.5 months \\
9 & $\mathrm{f}$ & 5 months \\
10 & $\mathrm{~m}$ & 6 months \\
11 & $\mathrm{f}$ & 2 months \\
12 & $\mathrm{~m}$ & 3 weeks \\
13 & $\mathrm{~m}$ & 9 months \\
14 & $\mathrm{~m}$ & 4 months \\
15 & $\mathrm{f}$ & 6 weeks \\
16 & $\mathrm{~m}$ & 6.5 months \\
17 & $\mathrm{~m}$ & 4 months \\
18 & $\mathrm{~m}$ & 3 months \\
19 & $\mathrm{f}$ & 2.5 months \\
20 & $\mathrm{f}$ & 3 months \\
21 & $\mathrm{~m}$ & 13 days \\
22 & $\mathrm{f}$ & 2 months \\
23 & $\mathrm{f}$ & 5 weeks \\
24 & $\mathrm{f}$ & 9 months \\
25 & $\mathrm{~m}$ & 3 months \\
26 & $\mathrm{~m}$ & 2 months \\
27 & $\mathrm{~m}$ & 4 months \\
28 & $\mathrm{~m}$ & 2 months \\
29 & $\mathrm{f}$ & 2.5 months \\
30 & $\mathrm{~m}$ & 7.5 months \\
31 & $\mathrm{~m}$ & 4 weeks \\
\hline & & \\
\hline & &
\end{tabular}

$-20{ }^{\circ} \mathrm{C}$. The following criteria applied: (1) sudden unexplained death of an infant $<1$ year of age, (2) no conclusive results of autopsy, (3) negative toxicology analysis, and (4) no pathological microscopic findings, except signs of cardiomyopathy. The study was approved by the local Ethic committee (protocol number E84/06).

Targeted sequencing Genomic DNA was isolated from stored tissue samples using phenol-chloroform extraction. The quality and quantity of DNA was assayed using the Nanodrop® ND-1000Spectromphotometer v3.1.0 (Thermo Fisher Scientific) and the Qubit Fluorometer 3.0 with dsDNA BR and HS assay kits (Invitrogen, Thermo Fisher Scientific), respectively. DNA integrity was assessed using the genomic DNA ScreenTape and Agilent 4200 TapeStation system.

Paired-end libraries were prepared following the manufacturer's protocol Nextera ${ }^{\mathrm{TM}}$ Flex for Enrichment (Illumina), and the TruSight cardio panel (Illumina) consisting of 174 genes with known cardiac associations was applied. Since the samples had been stored for a long period of time at $-20{ }^{\circ} \mathrm{C}$ and some samples showed DNA degradation, the recommendations for formalin-fixed paraffin-embedded (FFPE) samples in the protocol were followed. For samples exhibiting a DNA integrity number (DIN) lower than 5, $300 \mathrm{ng}$ of DNA was used as input for tagmentation, above a DIN of 5, 200 ng was used.

In order to obtain a better performance in GC-rich regions, the PCR program "amplify tagmented DNA" was modified and denaturation times in steps 2 and 3 were prolonged (4 min and $30 \mathrm{~s}$ instead of $3 \mathrm{~min}$ and $30 \mathrm{~s}$, respectively). Hybridization of probes was performed overnight. Enriched libraries were amplified with adjusted doubled denaturation times in steps 1 and 2 of the protocol. The genomic DNA sample NA12878 (Coriell Institute) was used as a control in each sequencing run.

Sequencing was performed on the Illumina ${ }^{\circledR}$ MiniSeq $^{\mathrm{TM}}$ system.

Variant analysis Resulting reads were aligned to the GRChr37 (hg19) human reference genome. GensearchNGS software (Phenosystems $®$ ) was used for evaluation of the data. The genetic data were filtered for aberrations in genes associated with cardiac channelopathies and cardiomyopathies $(n=80$, supplemental data) and according to a pre-established prioritization protocol mainly based on presumed functional impact on the protein and allele frequency. The resulting data were evaluated bioinformatically, and detected sequence variants were assessed using common databases (The Genome Aggregation Database, NHLBI Exome Sequencing Project, NCBI dbSNP, Human Gene Mutation Database) and applying in silico prediction tools (PolyPhen-2 [16], MutationTaster [17], SIFT [18], and CADD [19]).

Furthermore, two different minor allele frequencies (MAFs) were applied and the results compared. The MAF is the second most frequent allele value and is used to distinguish common 
polymorphisms from rare variants. The MAF of $0.2 \%$ reflects the prevalence of hypertrophic cardiomyopathy (HCM), known to be responsible for some SIDS cases. The MAF of $0.005 \%$ (1:20000 alleles) was used to evaluate "ultra-rare" variants based on a recent genetic SIDS-study [2]. For this evaluation, the minor allele frequency data of the Genome Aggregation Database (gnomAD) were utilized.

Filtered rare variants were classified according to the standards of the American College of Medical Genetics and Genomics (ACMG) [20]. Genetic variants expected to disrupt protein function (nonsense, frameshift, splice site variants, missense variants with established functional evidence) or rare missense variants within the core genes (supplemental data) were considered to be important variants (VUS, potentially informative), when they were located in a functional important domain, a mutation hotspot or described in this context in the literature.

\section{Results}

SIDS cohort Targeted sequencing was performed in 31 SIDS cases (18 male, 13 females; average age 3.4 months), which were collected as a part of the published German study on SIDS (GeSID) in the years from 1999 to 2001. The present study showed a higher risk of SIDS at the age of 2 to 3 months $(58 \%)$. There were no cases of SIDS at the age of 10 to 12 month (Fig. 1). The data represent a higher risk of SIDS in males $(58 \%)$, but gender distribution is equal at the age of 2-3 months.

Sequencing results Next-generation sequencing was successfully performed for all 31 SIDS cases with an average depth of coverage of $490 x$. For stringent variant classification, a standardized protocol was used. Filtering with a MAF of $\leq 0.2 \%$, a total of 14 rare variants were identified. After filtering with a MAF of $\leq 0.005 \%$, only 8 ultra-rare sequence variations were found. The potential significance of all sequence variations was assessed and classified according to the ACMG standards. Variants classified as uncertain significance were further divided in subclasses (VUS, probably benign and VUS, potentially informative) to better evaluate their potential relevance.

Rare variants were detected in 9 and ultra-rare variants in 8 of the 80 genes, respectively (Table 2). The distribution of these subclasses between the different MAFs is shown in Fig. 2.

By applying a MAF of $0.2 \%, 13(41 \%)$ cases with 14 rare variants were identified, with a MAF of $0.005 \%$, only $7(22 \%)$ cases with 8 ultra-rare variants were obtained. For variants considered to be "potentially informative" $(6 \%)$, no differences between the two (MAFs) allele frequency filters were observed. Moreover, with MAF of $0.005 \%$, in $16 \%$ variants were classified in a non-informative context; with MAF of $\leq$ $0.2 \%$, these variants were classified to exhibit uncertain significance (19\% instead of $16 \%$ ) or as benign (16\%).

\section{Genetic findings}

In case 1 , one potentially informative variant $\mathrm{p} .(\mathrm{Arg} 1316 \mathrm{Gln})$ was identified in the SCN5A-gene. In this case, no abnormalities of the heart have been found at autopsy and no cardiac disease history was reported by the family. The twin brother of the deceased infant exhibited no pathological cardiac symptoms. Abnormalities in the gene SCN5A are mainly associated with BrS and LQTS [21]. The detected variant is located at the transition site of the sodium channel in the fourth segment of the third domain close to the cytoplasm and has not been described in the literature and functional studies so far. In the database ClinVar the sequence variant was reported once in conjunction with $\mathrm{BrS}$ and was categorized as VUS. However, this variant may have functionally important, deleterious effects. The CADD-Score of 24.8 indicates that the nucleotide change may be of functional relevance. However, according to the ACMG guidelines, the potentially
Fig. 1 Occurrence of sudden infant's death within the first year of life

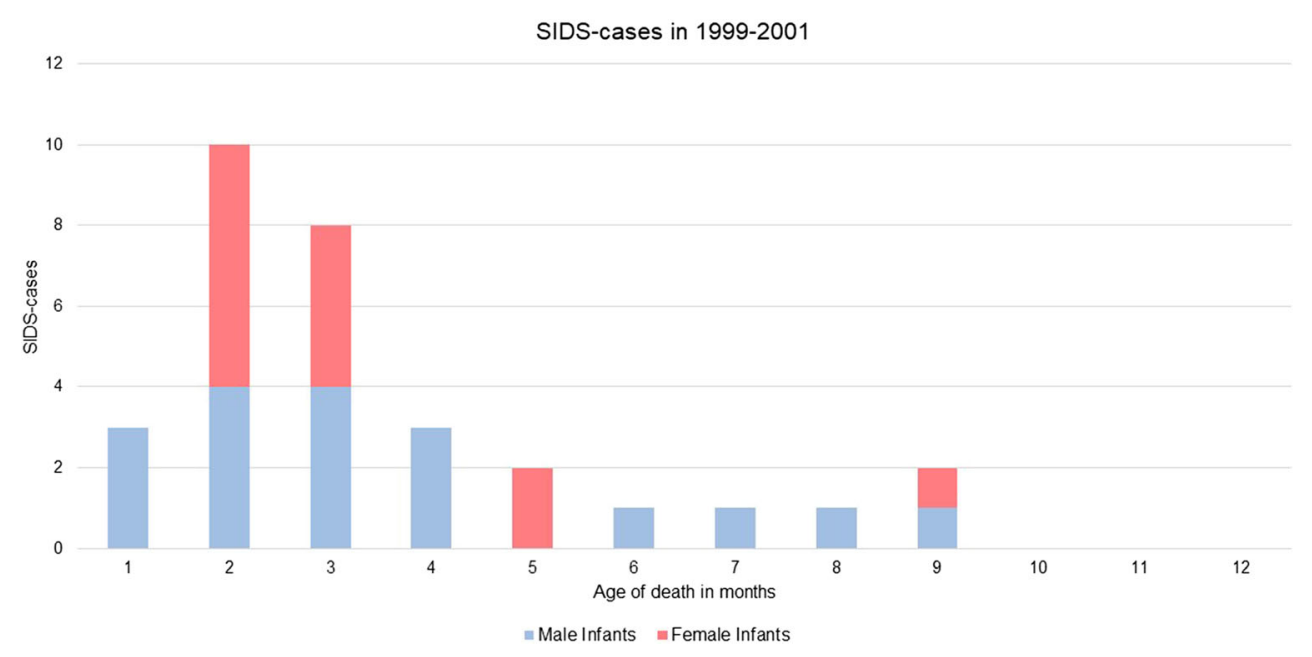


Table 2 Summary of variants identified in sudden infant death syndrome cases

\begin{tabular}{|c|c|c|c|c|c|c|c|c|c|c|c|}
\hline $\begin{array}{l}\text { SIDS } \\
\text { case }\end{array}$ & Age & Sex & Gene & Nucleotide change & $\begin{array}{l}\text { Amino acid } \\
\text { change }\end{array}$ & CADD & Polyphen & SIFT & MutationTaster & $\begin{array}{l}\text { MAF } \\
(\%)\end{array}$ & $\mathrm{ACMG}$ \\
\hline$\# 1$ & 7 weeks & $\mathrm{m}$ & SCN5A & c.3947 G > A (NM_198056) & p.(Arg1316GIn) & 24.8 & $\mathrm{PoD}$ & $\mathrm{D}$ & Disease causing & 0.003 & VUS \\
\hline \#2 & 2.5 months & $\mathrm{m}$ & RBM20 & $\begin{array}{l}\text { c.3595 G > A }\left(\mathrm{NM}_{-}^{-}\right. \\
001134363)\end{array}$ & p.(G1u1199Lys) & 23.4 & $\mathrm{~B}$ & $\mathrm{D}$ & Disease causing & 0.003 & VUS \\
\hline$\# 6$ & 1.5 months & $\mathrm{m}$ & CSRP3 & c.149 C > A (NM_003476) & p.(Ala50Glu) & 24.3 & $\operatorname{PrD}$ & $\mathrm{D}$ & Disease causing & 0.0007 & VUS \\
\hline \#7 & 2 months & $\mathrm{f}$ & MYH6 & c. $4505 \mathrm{G}>\mathrm{A}\left(\mathrm{NM} \_\right.$_002471) & p.(Arg1502GIn) & 32 & $\operatorname{PrD}$ & $\mathrm{D}$ & Disease causing & 0.016 & VUS \\
\hline$\# 15$ & 6 weeks & $\mathrm{f}$ & JUP & c. $1130 \mathrm{G}>\mathrm{A}\left(\mathrm{NM} \_002230\right)$ & p.(Arg377His) & 29.8 & $\operatorname{PrD}$ & $\mathrm{D}$ & Disease causing & 0.005 & VUS \\
\hline \multirow[t]{2}{*}{$\# 19$} & 2.5 months & $\mathrm{f}$ & MYH7 & c.4075 C > T (NM_000257) & p.(Arg1359Cys) & 29.5 & $\operatorname{PrD}$ & $\mathrm{D}$ & Disease causing & 0.0008 & VUS \\
\hline & & & $\mathrm{KCNJ} 2$ & c. 47 A > G (NM_000891) & p.(Glu16Gly) & 28.7 & $\mathrm{PoD}$ & $\mathrm{D}$ & Disease causing & 0.0004 & VUS \\
\hline \#22 & 2 months & $\mathrm{f}$ & PRKAG2 & c. 1508 A > G (NM_016203) & p.(GIn503Arg) & 25.5 & $\mathrm{PoD}$ & $\mathrm{T}$ & Disease causing & 0.001 & VUS \\
\hline \#21 & 4 months & $\mathrm{m}$ & LMNA & c.632 A > G (NM_170707) & p.(Tyr211Cys) & 24.6 & $\mathrm{PoD}$ & $\mathrm{T}$ & Disease causing & 0.003 & VUS \\
\hline
\end{tabular}

informative variant has to be classified as variant of uncertain significance due to missing functional characterization studies as well as co-segregation data.

In case 19 no pathological abnormalities of the heart had been detected at autopsy. According to the family history, another SIDS case had occurred in the year before. One variant, p.(Arg1359Cys), was detected in the MYH7-gene, which has been described to be associated with cardiomyopathy. In ClinVar the variant is categorized as variant of uncertain significance. The high CADD-Score (29.5) suggests a pathogenic effect. The variant was classified as potentially informative VUS based on the criteria as shown above. According to Hershberger et al. [22] and Klaassen et al. [23], this variant has been found in patients with cardiomyopathy and is considered to be potentially pathogenic.

The second variant in this case p.(Glu16Gly) was detected in the KCNJ2-gene. This gene is associated with LQTS,
Fig. 2 Percentage of variants associated with genetic heart diseases in SIDS cases. A With a minor allele frequency (MAF) $\leq$ $0.2 \%$. B With a minor allele frequency $(\mathrm{MAF}) \leq 0.005 \%$
SQTS, and CPVT [24, 25], and the detected variant has not been described in ClinVar so far. The high CADD-Score (28.7) suggests pathogenicity. Based on the ACMG guidelines, the variant was classified as VUS, since the variant is not localized in a functional important domain and no cosegregation data are reported or functional studies exist.

Following the strict ACMG guidelines and a conservative MAF, 2 of the ultra-rare variants present in two (6\%) of the 31 SIDS cases were classified as VUS with potentially pathogenic impact.

\section{Discussion}

Although the incidence of the sudden infant death syndrome (SIDS) drastically decreased during the last years, it is still one of the leading causes of death in industrialized countries [5].

\section{Variants in SIDS-cases}

(a)

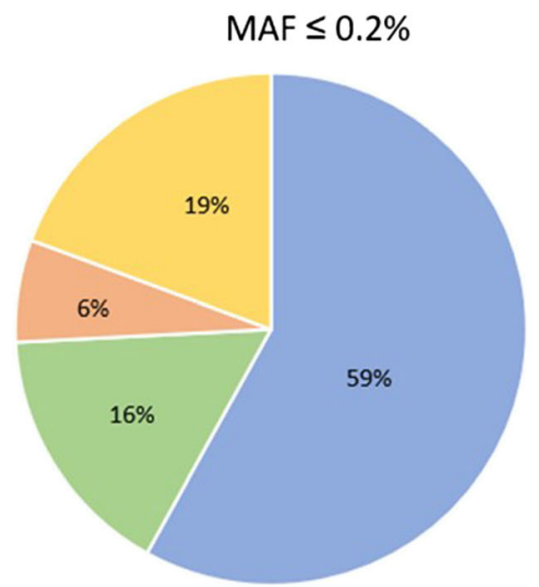

(b)

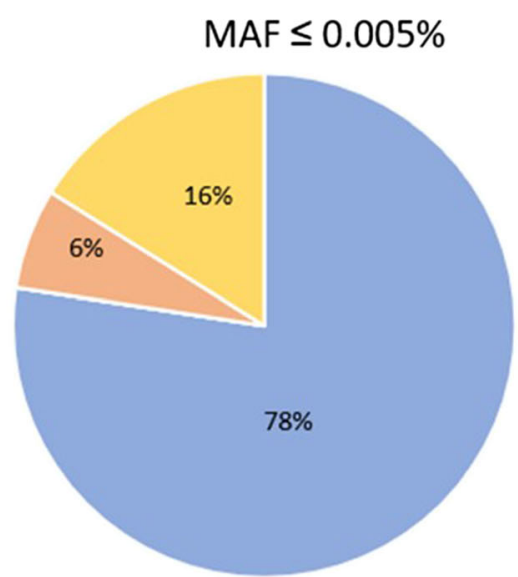

- SIDS-cases without informative variants $=$ VUS probably benign - VUS potentially informative VUS uncertain 
SIDS is a multifactorial and heterogeneous incident, which may be associated with variants in various genes.

Inherited channelopathies as well as cardiomyopathies have been considered as a monogenic cause in some SIDS cases [2]. In studies of the genetic background of SIDS victims next-generation sequencing technologies represent a novel approach [26-28].

In the present study, postmortem genetic screening of 80 candidate genes was performed in selected SIDS cases in order to detect the presence of potentially causative variants in a spectrum of cardiac arrhythmia syndrome-related genes. The study included a cohort of 31 SIDS infants with a peak incidence between 2 and 4 months of age and slightly higher prevalence in boys (58\%), which is in line with previous studies $[2,3,5]$.

Although the study confirms the high potential of NGS to identify informative variants, a careful and stringent variant analysis is essential to enable appropriate counseling of the families involved.

Therefore, evaluating the sequencing data using a threshold for allele frequency is one of the most important criteria in an accurate data analysis. Since prevalence of SIDS is 1:5000 in live births and causative disorders are rare, two different MAF cutoffs were used in the present study: $0.2 \%$, which is based on the prevalence of hypertrophic cardiomyopathy (1:500) and $0.005 \%$ ( 1 in 20,000 alleles or 1 in 10,000 individuals), reflecting rare variants as recommended in a study by Tester et al. [2]. By applying these two allele frequency filters, we tried to minimize the problem of incorrect over-interpretation of presumably pathogenic variants and the possibility of excluding potentially pathogenic variants that are rare. Using this strategy, $25 \%$ of cohort members possessed a rare genetic variant. In two cases (6\%), the variants were classified as potentially pathogenic variants of uncertain significance. However, despite their rarity, the pathogenic significance of the variants is still unknown. Neubauer et al. [4] detected variants with potentially pathogenic effects in $20 \%$, Hertz et al. [29] in 34\% of SIDS cases, respectively. In both studies, a relatively high allele frequency of $<1 \%$ for filtering of variants was used. However, these rather high values in the assessment of pathogenic variants are probably overestimating their relevance in respect of the rarity SIDS represents. The data of the present study rather support those obtained by Tester et al. [2], although the frequency of informative variants is significantly lower. The lower allele frequency of $0.005 \%$ leads to potentially informative variants in only $2(6 \%)$ out of 31 cases. These differences clearly show the need of a standardized practice for the interpretation of rare variants associated with inherited diseases.

Allele frequency The comparison of probably benign variants indicates that they are omitted by lower allele frequency and therefore are not rare. Based on previous results, filtering with a low allele frequency is useful for assessing variants, but one has to keep in mind that potentially important variants and information with regard to genetic modifiers may be missed, which could play a role in SIDS, but are too common to be detected with the filter applied.

Study limitations Based on the size of the gene Titin (TTN) and the high number of missense variants with limited clinical value, detected missense TTN variants were not further assessed.

Since the samples had been collected anonymously, the families related with the cases exhibiting probably informative variants could not be informed. Therefore, important results of cardiological and genetic investigations of family members for co-segregation studies were not possible.

\section{Conclusion}

Targeted sequencing using a cardiac gene-specific focus reveals that about $20 \%$ of 31 SIDS cases exhibited a variant of uncertain significance. By applying a stringent variant classification, only $6 \%$ of these cases showed a potentially pathogenic variant. The study clearly points to the importance of careful variant interpretation. Results of genetic analyses may have an important impact on the family members involved.

Acknowledgements Open Access funding provided by Projekt DEAL.

Funding information We thank the Friedrich Flick Foundation for supporting this work.

\section{Compliance with ethical standards}

Ethical approval All procedures performed in studies involving human participants were in accordance with the ethical standards of the institutional and/or national research committee and with the 1964 Helsinki declaration and its later amendments or comparable ethical standards.

Open Access This article is licensed under a Creative Commons Attribution 4.0 International License, which permits use, sharing, adaptation, distribution and reproduction in any medium or format, as long as you give appropriate credit to the original author(s) and the source, provide a link to the Creative Commons licence, and indicate if changes were made. The images or other third party material in this article are included in the article's Creative Commons licence, unless indicated otherwise in a credit line to the material. If material is not included in the article's Creative Commons licence and your intended use is not permitted by statutory regulation or exceeds the permitted use, you will need to obtain permission directly from the copyright holder. To view a copy of this licence, visit http://creativecommons.org/licenses/by/4.0/.

\section{References}

1. Psaila K, Foster JP, Pulbrook N, Jeffery HE, Cochrane Neonatal Group (2017) Infant pacifiers for reduction in risk of sudden infant death syndrome. Cochrane Database Syst Rev 4:CD011147. https://doi.org/10.1002/14651858.CD011147.pub2 
2. Tester DJ, Wong LCH, Chanana P, Jaye A, Evans JM, FitzPatrick DR, Evans MJ, Fleming P, Jeffrey I, Cohen MC, Tfelt-Hansen J, Simpson MA, Behr ER, Ackerman MJ (2018) Cardiac genetic predisposition in sudden infant death syndrome. J Am Coll Cardiol 71(11):1217-1227. https://doi.org/10.1016/j.jacc.2018.01.030

3. Möllborg P, Alm B (2010) Sudden infant death syndrome during low incidence in Sweden 1997-2005. Acta Paediatr 99(1):94-98. https://doi.org/10.1111/j.1651-2227.2009.01539.x

4. Neubauer J, Lecca MR, Russo G, Bartsch C, Medeiros-Domingo A, Berger W, Haas C (2017) Post-mortem whole-exome analysis in a large sudden infant death syndrome cohort with a focus on cardiovascular and metabolic genetic diseases. Eur J Hum Genet 25(4):404-409. https://doi.org/10.1038/ejhg.2016.199

5. Moon RY, Horne RSC, Hauck FR (2007) Sudden infant death syndrome. Lancet 370(9598):1578-1587. https://doi.org/10.1016/ S0140-6736(07)61662-6

6. Ackerman MJ, Siu BL, Sturner WQ, Tester DJ, Valdivia CR, Makielski JC, Towbin JA (2001) Postmortem molecular analysis of SCN5A defects in sudden infant death syndrome. JAMA 286(18):2264-2269. https://doi.org/10.1001/jama.286.18.2264

7. Kinney HC, Richerson GB, Dymecki SM, Darnall RA, Nattie EE (2009) The brainstem and serotonin in the sudden infant death syndrome. Annu Rev Pathol 4:517-550. https://doi.org/10.1146/ annurev.pathol.4.110807.092322

8. Weese-Mayer DE, Ackerman MJ, Marazita ML, Berry-Kravis EM (2007) Sudden infant death syndrome: review of implicated genetic factors. Am J Med Genet A 143A(8):771-788. https://doi.org/10. 1002/ajmg.a.31722

9. van Norstrand DW, Ackerman MJ (2010) Genomic risk factors in sudden infant death syndrome. Genome Med 2(11):86. https://doi. org/10.1186/gm207

10. Schwartz PJ, Stramba-Badiale M, Segantini A, Austoni P, Bosi G, Giorgetti R, Grancini F, Marni ED, Perticone F, Rosti D, Salice P (1998) Prolongation of the QT interval and the sudden infant death syndrome. N Engl J Med 338(24):1709-1714. https://doi.org/10. 1056/NEJM199806113382401

11. Cronk LB, Ye B, Kaku T, Tester DJ, Vatta M, Makielski JC, Ackerman MJ (2007) Novel mechanism for sudden infant death syndrome: persistent late sodium current secondary to mutations in caveolin-3. Heart Rhythm 4(2):161-166. https://doi.org/10. 1016/j.hrthm.2006.11.030

12. Tester DJ, Ackerman MJ (2005) Sudden infant death syndrome: how significant are the cardiac channelopathies? Cardiovasc Res 67(3):388-396. https://doi.org/10.1016/j.cardiores.2005.02.013

13. Tester DJ, Dura M, Carturan E, Reiken S, Wronska A, Marks AR, Ackerman MJ (2007) A mechanism for sudden infant death syndrome (SIDS): stress-induced leak via ryanodine receptors. Heart Rhythm 4(6):733-739. https://doi.org/10.1016/j.hrthm.2007.02. 026

14. Brion M, Allegue C, Santori M, Gil R, Blanco-Verea A, Haas C, Bartsch C, Poster S, Madea B, Campuzano O, Brugada R, Carracedo A (2012) Sarcomeric gene mutations in sudden infant death syndrome (SIDS). Forensic Sci Int 219(1-3):278-281. https://doi.org/10.1016/j.forsciint.2012.01.018

15. Findeisen M, Vennemann M, Brinkmann B et al (2004) German study on sudden infant death (GeSID): design, epidemiological and pathological profile. Int J Legal Med 118(3):163-169. https://doi. org/10.1007/s00414-004-0433-8

16. Adzhubei IA, Schmidt S, Peshkin L, Ramensky VE, Gerasimova A, Bork P, Kondrashov AS, Sunyaev SR (2010) A method and server for predicting damaging missense mutations. Nat Methods 7(4):248-249. https://doi.org/10.1038/nmeth0410-248

17. Schwarz JM, Cooper DN, Schuelke M, Seelow D (2014) MutationTaster2: mutation prediction for the deep-sequencing age. Nat Methods 11(4):361-362. https://doi.org/10.1038/nmeth. 2890

18. Sim N-L, Kumar P, Hu J et al (2012) SIFT web server: predicting effects of amino acid substitutions on proteins. Nucleic Acids Res 40(Web Server issue):W452-W457. https://doi.org/10.1093/nar/ gks539

19. Rentzsch P, Witten D, Cooper GM, Shendure J, Kircher M (2019) CADD: predicting the deleteriousness of variants throughout the human genome. Nucleic Acids Res 47(D1):D886-D894. https:// doi.org/10.1093/nar/gky1016

20. Richards S, Aziz N, Bale S et al (2015) Standards and guidelines for the interpretation of sequence variants: a joint consensus recommendation of the American College of Medical Genetics and Genomics and the Association for Molecular Pathology. Genet Med 17(5):405-424. https://doi.org/10.1038/gim.2015.30

21. Daimi H, Khelil AH, Neji A, Ben Hamda K, Maaoui S, Aranega A, BE. Chibani J, Franco D (2019) Role of SCN5A coding and noncoding sequences in Brugada syndrome onset: What's behind the scenes? Biom J 42(4):252-260. https://doi.org/10.1016/j.bj.2019. 03.003

22. Hershberger RE, Parks SB, Kushner JD, Li D, Ludwigsen S, Jakobs P, Nauman D, Burgess D, Partain J, Litt M (2008) Coding sequence mutations identified in MYH7, TNNT2, SCN5A, CSRP3, LBD3, and TCAP from 313 patients with familial or idiopathic dilated cardiomyopathy. Clin Transl Sci 1(1):21-26. https://doi.org/10. 1111/j.1752-8062.2008.00017.x

23. Klaassen S, Probst S, Oechslin E, Gerull B, Krings G, Schuler P, Greutmann M, Hürlimann D, Yegitbasi M, Pons L, Gramlich M, Drenckhahn J̈D, Heuser A, Berger F, Jenni R, Thierfelder L (2008) Mutations in sarcomere protein genes in left ventricular noncompaction. Circulation 117(22):2893-2901. https://doi.org/ 10.1161/CIRCULATIONAHA.107.746164

24. Napolitano C, Bloise R, Monteforte N, Priori SG (2012) Sudden cardiac death and genetic ion channelopathies: long QT, Brugada, short QT, catecholaminergic polymorphic ventricular tachycardia, and idiopathic ventricular fibrillation. Circulation 125(16):20272034. https://doi.org/10.1161/CIRCULATIONAHA.111.055947

25. Kalscheur MM, Vaidyanathan R, Orland KM, Abozeid S, Fabry N, Maginot KR, January CT, Makielski JC, Eckhardt LL (2014) $\mathrm{KCNJ} 2$ mutation causes an adrenergic-dependent rectification abnormality with calcium sensitivity and ventricular arrhythmia. Heart Rhythm 11(5):885-894. https://doi.org/10.1016/j.hrthm. 2014.02.015

26. Bezzina CR, Lahrouchi N, Priori SG (2015) Genetics of sudden cardiac death. Circ Res 116(12):1919-1936. https://doi.org/10. 1161/CIRCRESAHA.116.304030

27. Brion M, Sobrino B, Martinez M, Blanco-Verea A, Carracedo A (2015) Massive parallel sequencing applied to the molecular autopsy in sudden cardiac death in the young. Forensic Sci Int Genet 18: 160-170. https://doi.org/10.1016/j.fsigen.2015.07.010

28. Bagnall RD, Semsarian C (2017) Role of the molecular autopsy in the investigation of sudden cardiac death. Prog Pediatr Cardiol 45: 17-23. https://doi.org/10.1016/j.ppedcard.2017.02.005

29. Hertz CL, Christiansen SL, Larsen MK, Dahl M, Ferrero-Miliani L, Weeke PE, Pedersen O, Hansen T, Grarup N, Ottesen GL, FrankHansen R, Banner J, Morling N (2016) Genetic investigations of sudden unexpected deaths in infancy using next-generation sequencing of 100 genes associated with cardiac diseases. Eur J Hum Genet 24(6):817-822. https://doi.org/10.1038/ejhg.2015.198

Publisher's note Springer Nature remains neutral with regard to jurisdictional claims in published maps and institutional affiliations. 